\title{
Efeito da densidade de estocagem no desenvolvimento inicial do acará-bandeira (Pterophyllum scalare)
}

\author{
[The effect of storage density in the early development of angelfish (Pterophyllum scalare)] \\ L.P. Gonçalves Júnior, S.L. Pereira, M.D. Matielo, P.P. Mendonça \\ Instituto Federal do Espírito Santo - IFES - Alegre, ES
}

\begin{abstract}
RESUMO
Avaliou-se a influência da densidade de estocagem no desenvolvimento inicial do acará-bandeira (Pterophyllum scalare). O desempenho produtivo foi avaliado pelo ganho de peso (GP), ganho de comprimento total (GCT), ganho de comprimento padrão (GCP), ganho de altura (GA), taxa de crescimento específico (TCE), taxa de desenvolvimento específico (TDE) e sobrevivência (S). Para realização do experimento, foram utilizadas 300 pós-larvas de peso médio inicial de 0,0012g, comprimento total médio de $4,11 \pm 0,93 \mathrm{~mm}$, altura média inicial de $4,84 \pm 0,87 \mathrm{~mm}$ e comprimento padrão médio de 1,54 $\pm 0,25 \mathrm{~mm}$. Essas foram distribuídas em delineamento inteiramente ao acaso, em cinco tratamentos com quatro repetições. Os tratamentos $(\mathrm{T})$ foram constituídos por cinco diferentes densidades de estocagem, em que T1: 5 pós-larvas $\mathrm{L}^{-1}$; T2: 10 pós-larvas $\mathrm{L}^{-1}$; T3: 15 pós-larvas $\mathrm{L}^{-1}$; T4: 20 pós-larvas $\mathrm{L}^{-1}$; T5: 25 pós-larvas $\mathrm{L}^{-1}$. A densidade de 5 pós-larvas $\mathrm{L}^{-1}$ foi a que proporcionou o maior valor para crescimento. Contudo, essa densidade subestimou a utilização do espaço se comparada com a produtividade final da densidade de 15 pós-larvas $\mathrm{L}^{-1}$.
\end{abstract}

Palavras-chave: peixe ornamental, larvicultura, intensificação do cultivo

\begin{abstract}
The influence of stocking density in the initial development of angelfish (Pterophyllum scalare) was evaluated. The productive performance was evaluated by weight gain (WG) gain length (GCT), standard length gain (GCP), height gain (GH), specific growth rate (TCE), specific development rate (TDE) and survival (S). For the experiment we used a total of 300 post-larvae angelfish with initial body weight of $0.0012 \mathrm{~g}$, total length of $4.11 \pm 0.93 \mathrm{~mm}$, initial height of $4.84 \pm 0.87 \mathrm{~mm}$, and average standard length of $1.54 \pm 0.25 \mathrm{~mm}$. These were distributed in a completely randomized design in five treatments with four replications. The treatments consisted of five different stocking densities where T1: 5 post-larvae $L^{-1}$, T2: 10 post-larvae $L^{-1}$, T3: 15 post-larvae $L^{-1}$, T4: 20 post-larvae $L^{-1}$, T5: 25 post-larvae $L^{-1}$. The physical and chemical water parameters were monitored daily. The density of post-larvae $5 L^{-1}$ was the one that gave the highest growth values. However, despite the post-larvae $5 L^{-1}$ density having provided larger animals, the density underestimates the use of space compared to the final productivity of the 15 post-larvae $L^{-}$ ${ }^{1}$ density.
\end{abstract}

Keywords: ornamental fish, hatchery, intensification of cultivation

\section{INTRODUÇÃO}

O mercado de espécies ornamentais é um segmento importante na aquicultura mundial e vem demostrando grande crescimento nos últimos anos. Segundo Ribeiro (2008), de 2002 até 2006 as exportações de peixes ornamentais

Recebido em 23 de novembro de 2011

Aceito em 12 de dezembro de 2012

E-mail: juniorvezula@hotmail.com cresceram, em média, $11,6 \%$ ao ano, gerando um lucro de mais de USD 277,0 milhões em 2006. O Brasil é um dos grandes fornecedores mundiais de espécies ornamentais, destacando-se o estado de Minas Gerais como um dos maiores centros. Nessa região, há um grande número de produtores dedicados à produção de mais de 50 espécies, tais como o acará-disco 
(Symphysodon discus), guppy (Poecilia reticulata), tetra neon (Paracheirodon innesi), beta (Betta splendens), cardinal (Paracheirodon axeroldi), inclusive à criação do acará-bandeira (Pterophyllum scalare) (Pezzato e Scorvo Filho, 2000).

O acará-bandeira (Pterophyllum scalare), pertencente à família dos ciclídeos, originária da bacia Amazônica, encontra-se amplamente distribuído no Peru, Colômbia, Guianas e Brasil (Cacho et al., 1999). Destaca-se pela convivência pacífica com inúmeras outras espécies. Apresenta grande aceitabilidade no mercado por ser um dos mais belos, mais vendidos e, também, mais populares peixes de águas tropicais, estando entre as oito espécies de peixes ornamentais mais comercializadas mundialmente (Chapman et al., 1997). Há algumas variedades de acará-bandeira, como marmorato, ouro, koi, leopardo, preto, fumaça e palhaço, que se adaptam bem ao manejo nos mais diversos sistemas de cultivo, apresentando valor comercial até 10 vezes superior aos exemplares capturados da natureza (Ribeiro, 2009).

Os sistemas de produção animal demonstram uma tendência de intensificação do cultivo. Um dos principais motivos para se justificarem os esforços voltados para a intensificação dos sistemas de produção animal está relacionado à melhor remuneração do capital, à melhor utilização dos recursos e ao consequente aumento da produtividade (Berlli, 2010). Entretanto, um dos maiores problemas enfrentados na produção de espécies ornamentais está relacionado ao início de seu desenvolvimento, em que seu potencial produtivo não é explorado corretamente, pois são poucas as informações disponíveis. Portanto, o manejo desses peixes tem como base as informações padronizadas para espécies exóticas, principalmente da piscicultura de corte (Takahashi et al., 2010).

Assim, a expansão do setor produtivo de peixes ornamentais e o consequente suprimento dos mercados interno e externo dependem de informações relacionadas ao potencial zootécnico de cada espécie cultivada. Embora tal conhecimento seja considerado de grande importância no sucesso da atividade, raros são os estudos voltados para o início do desenvolvimento. Assim, o objetivo do presente trabalho foi avaliar a influência da densidade de estocagem no desenvolvimento inicial do acarábandeira (Pterophyllum scalare).

\section{MATERIAL E MÉTODOS}

O presente estudo foi realizado entre os dias 26 de março e 25 de abril de 2011, no Laboratório de Pesquisas com Espécies Ornamentais na Seção de Aquicultura do Instituto Federal do Espírito Santo, Campus de Alegre. As unidades experimentais foram compostas por recipientes plásticos com volume útil de $1 \mathrm{~L}$, sem a presença de aeração. Para realização do experimento, foi utilizado um total de 300 pós-larvas recémeclodidas, de peso médio inicial de $0,0012 \mathrm{~g}$, comprimento total médio de $4,11 \pm 0,93 \mathrm{~mm}$, altura média inicial de $4,84 \pm 0,87 \mathrm{~mm}$ e comprimento padrão médio de $1,54 \pm 0,25 \mathrm{~mm}$.

Em relação à biometria inicial, utilizou-se um microscópio estereoscópico com lente macrométrica, onde foi verificado o tamanho inicial dos animais. Para estimar o peso médio inicial, devido ao pequeno tamanho e à fragilidade dos animais utilizados, foi feita a pesagem em grupo de 10 exemplares em uma balança analítica com precisão de $0,0001 \mathrm{mg}$ e, a partir do valor encontrado, obteve-se o peso médio inicial. Para montagem do experimento, as pós-larvas foram coletas aleatoriamente de um mesmo lote e distribuídas em delineamento inteiramente ao acaso, em cinco tratamentos ( $\mathrm{T}$ ) com quatro repetições. Os tratamentos foram constituídos por cinco diferentes densidades de estocagem, em que T1: 5 pós-larvas $\mathrm{L}^{-1}$; T2: 10 pós-larvas $\mathrm{L}^{-1}$; T3: 15 pós-larvas $\mathrm{L}^{-1}$; T4: 20 póslarvas $\mathrm{L}^{-1}$; T5: 25 pós-larvas $\mathrm{L}^{-1}$.

Para alimentação, foram preparadas incubadoras com capacidade de $2 \mathrm{~L}$ d'água para a eclosão de náuplios de Artêmia sp.; $2 \mathrm{~g}$ de Artêmia sp. e salinidade de quatro partes por mil combinadas com uma temperatura de $28^{\circ} \mathrm{C}$, condição suficiente para a eclosão dos náuplios de Artêmia sp. após 24 horas. Passado esse período, os náuplios foram separados dos cistos e fornecidos até a saciedade aparente como único alimento em todo período experimental, três vezes ao dia, às 7 h, 11 h30min e 16 horas.

Logo após a última alimentação, para a remoção das sobras de alimento e das fezes, diariamente se realizava o sifonamento de cada 
unidade experimental, com reposição de água correspondente a 30\% do volume total. Quando observada mortalidade, o animal morto era imediatamente retirado, e o volume da unidade experimental reajustado conforme o número de animais que ainda restavam no recipiente, sendo possível manter a mesma densidade de estocagem.

Os seguintes parâmetros físico-químicos da água foram mensurados diariamente durante o período experimental: oxigênio dissolvido ( $\mathrm{mg} / \mathrm{L}), \mathrm{pH}$, temperatura $\left({ }^{\circ} \mathrm{C}\right)$ e condutividade elétrica $(\mu \mathrm{S})$, sempre após a última alimentação do dia.

Ao final do $25^{\circ}$ dia, os animais foram mantidos em jejum por 12 horas e, após esse período, foi realizada a biometria final, com auxílio de um paquímetro e balança analítica com precisão de $0,0001 \mathrm{mg}$. Assim, foram verificados os valores individuais de peso $(\mathrm{mg})$, comprimento total $(\mathrm{mm})$, comprimento padrão $(\mathrm{mm})$ e altura $(\mathrm{mm})$ de cada unidade experimental, e também foi avaliada a sobrevivência (S).

O desempenho produtivo foi avaliado pelo ganho de peso (GP) calculado pela diferença do peso final e do peso inicial dos animais. Com a diferença entre os tamanhos inicial e final dos animais, foi possível estimar o ganho de comprimento total (GCT), ganho de comprimento padrão (GCP) e ganho de altura (GA). Foi calculada, também, a taxa de crescimento específico (TCE) e a taxa de desenvolvimento específico (TDE), essas duas últimas variáveis expressam a taxa de crescimento em peso e o comprimento dos animais, respectivamente, durante o período experimental.

Taxa de crescimento específico $=[(\log$ peso final - $\log$ peso inicial) $/ \mathrm{n}^{\mathrm{o}}$ de dias experimentais) ] $\mathrm{x}$ 100 .

Taxa de desenvolvimento específico $=[(\log \mathrm{CT}$ final $-\log$ CT inicial) $/ \mathrm{n}^{\circ}$ de dias experimentais)] x 100 .

Para cada variável mensurada no experimento, foi realizada uma ANOVA geral, para verificar a existência de diferença entre os tratamentos. Após a ANOVA, os resultados foram submetidos ao teste de Tukey $(\mathrm{P}<0,10)$. Todos os cálculos foram realizados com auxílio do programa estatístico SAEG 9.0.

\section{RESULTADOS E DISCUSSÃO}

Os parâmetros físico-químicos da água não apresentaram diferença significativa para as densidades testadas (Tab. 1).

Tabela 1. Valores médios \pm desvio-padrão para temperatura ( $\mathrm{T}$ ), pH, oxigênio dissolvido (OD) e condutividade elétrica (CE) nos diferentes tratamentos durante 25 dias de experimentação

\begin{tabular}{lccccc}
\hline \multirow{2}{*}{ Variável } & \multicolumn{5}{c}{ Número de peixes $\mathrm{L}^{-1}$} \\
\cline { 2 - 5 } & 5 & 10 & 15 & 20 & 25 \\
\hline $\mathrm{T}\left({ }^{\circ} \mathrm{C}\right)$ & $28,55 \pm 0,60$ & $28,60 \pm 0,56$ & $28,58 \pm 0,60$ & $28,57 \pm 0,58$ & $28,54 \pm 0,53$ \\
$\mathrm{Ph}$ & $7,7 \pm 0,3$ & $7,7 \pm 0,4$ & $7,7 \pm 0,4$ & $7,6 \pm 0,4$ & $7,6 \pm 0,4$ \\
$\mathrm{OD}(\mathrm{mg} / \mathrm{L})$ & $7,53 \pm 1,06$ & $7,43 \pm 0,78$ & $7,48 \pm 0,72$ & $7,68 \pm 1,7$ & $7,64 \pm 0,79$ \\
$\mathrm{CE}(\mu \mathrm{S})$ & $0,365 \pm 0,128$ & $0,299 \pm 0,098$ & $0,424 \pm 0,147$ & $0,403 \pm 0,119$ & $0,3370,113$ \\
\hline
\end{tabular}

Os parâmetros físico-químicos da água ficaram dentro do esperado para o adequado desenvolvimento do acará-bandeira (Cruz et al., 2002; Pérez et al., 2003). Esses autores recomendaram faixa de temperatura entre $26 \mathrm{e}$ $30^{\circ} \mathrm{C}$ e concentração de oxigênio dissolvido acima de $5 \mathrm{mg} / \mathrm{L}$ e ressaltaram que, apesar de originária de águas ácidas, essa espécie apresenta tolerância a variações de $\mathrm{pH}$ na faixa de 5,5 e 8, desde que a mudança ocorra gradativamente. A condutividade apresentou valores elevados, porém não afetou o desenvolvimento da espécie.
Os diferentes tratamentos demonstraram efeito significativo $(\mathrm{P}<0,10)$ sobre o tamanho e o peso final dos animais. Os valores médios referentes ao tamanho das pós-larvas de acará-bandeira após 25 dias de experimentação foram maiores para as menores densidades testadas (Tab. 2). Não foi observada influência da densidade de estocagem sobre a variável sobrevivência $(\mathrm{P}<0,10)$. Entretanto, o aumento da densidade afetou negativamente a taxa de sobrevivência. 
Tabela 2. Valores para peso final (PF), comprimento total final (CTF), comprimento padrão final (CPF), altura final (AF) e sobrevivência (SO) das pós-larvas de acará-bandeira após 25 dias de experimentação, submetidas a diferentes densidades

\begin{tabular}{cccccc}
\hline \multirow{2}{*}{ Variável } & \multicolumn{5}{c}{ Número de peixes $\mathrm{L}^{-1}$} \\
\cline { 2 - 6 } & 5 & 10 & 15 & 20 & 25 \\
\hline PF $(\mathrm{g})$ & $0,042 \mathrm{a}$ & $0,039 \mathrm{a}$ & $0,035 \mathrm{ab}$ & $0,022 \mathrm{bc}$ & $0,025 \mathrm{c}$ \\
CTF $(\mathrm{mm})$ & $16,00 \mathrm{a}$ & $14,27 \mathrm{ab}$ & $13,30 \mathrm{~b}$ & $12,68 \mathrm{~b}$ & $12,64 \mathrm{~b}$ \\
CPF $(\mathrm{mm})$ & $10,75 \mathrm{a}$ & $10,05 \mathrm{ab}$ & $10,00 \mathrm{ab}$ & $9,31 \mathrm{~b}$ & $9,38 \mathrm{~b}$ \\
AF $(\mathrm{mm})$ & $5,12 \mathrm{a}$ & $4,33 \mathrm{ab}$ & $4,75 \mathrm{ab}$ & $3,90 \mathrm{~b}$ & $4,40 \mathrm{ab}$ \\
SO $(\%)$ & $100 \mathrm{a}$ & $50 \mathrm{a}$ & $58 \mathrm{a}$ & $43 \mathrm{a}$ & $45 \mathrm{a}$ \\
\hline
\end{tabular}

Médias com letras diferentes na mesma linha diferem pelo teste de Tukey $(\mathrm{P}<0,10)$.

No presente trabalho, foi observada uma tendência de redução da sobrevivência com o aumento da densidade de estocagem. Os valores de sobrevivência encontrados são considerados baixos quando comparados com os de Chapman (2000), que relatou sobrevivência mínima de $85 \%$ para espécies ornamentais, de maneira geral. Porém, resultado similar para sobrevivência foi observado por Silva (2008), ao estudar a viabilidade do policultivo na fase inicial entre o camarão (Macrobrachium rosenbergii), acará-bandeira (Pterophyllum scalare) e o japonês (Carassius auratus). Foram encontrados valores de sobrevivência entre 30 e $87,5 \%$ para o acará-bandeira. As altas taxas de mortalidade podem estar associadas a condições extremas de estresse, em razão do aumento da densidade de estocagem. Segundo Luz (2007), esse efeito é devido ao aumento da competitividade entre os animais, ao aumento da deterioração da qualidade da água e, consequentemente, à maior susceptibilidade dos animais aos patógenos.

Os menores valores para peso final foram de 0,022 e $0,025 \mathrm{~g}$, obtidos para as densidades de 20 e 25 pós-larvas $\mathrm{L}^{-1}$, respectivamente, sendo menores $(\mathrm{P}>0,10)$ que $0,042 \mathrm{~g}$ obtido na densidade de 5 pós-larvas $\mathrm{L}^{-1}$. Luz e Zaniboni Filho (2002) encontraram efeito da densidade de estocagem no peso já nos primeiros cinco dias de cultivo. Esses autores submeteram pós-larvas de mandi-amarelo (Siluriformes: Pimelodidae) a diferentes densidades, mantidas em águas de $\mathrm{pH}$ 6 e faixas de temperatura que variaram entre $22,4 \pm 1,4$ e $29,6 \pm 1,7^{\circ} \mathrm{C}$, e verificaram que a densidade de 5 pós-larvas $\mathrm{L}^{-1}$ resultou em animais de peso médio final de $0,025 \mathrm{~g}$, sendo significativamente maior que o valor encontrado na densidade de 15 pós-larvas $\mathrm{L}^{-1}$, a qual resultou em animais de peso médio final de $0,021 \mathrm{~g}$.

Como o peso final foi maior nos tratamentos que utilizaram as densidades de 5, 10 e 15 pós-larvas $\mathrm{L}^{-1}$, o comprimento total final (CTF) e a altura final (AF) também foram maiores e diferiram dos tratamentos que submeteram os animais às densidades de 20 e 25 pós-larvas $\mathrm{L}^{-1}$.

O maior valor para comprimento padrão final de $10,75 \mathrm{~mm}$ foi obtido para os animais mantidos na densidade de 5 pós-larvas $\mathrm{L}^{-1}$. Entretanto, essa densidade subestima a utilização do espaço quando comparada à densidade de 15 pós-larvas $\mathrm{L}^{-1}$.

$\mathrm{Na}$ Tab. 3 encontra-se o desempenho produtivo das pós-larvas de acará-bandeira submetidas aos diferentes tratamentos.

Tabela 3. Valores para ganho de peso (GP), ganho de comprimento total (GCT), ganho de comprimento padrão (GCP), ganho de altura (GA), taxa de crescimento específico (TCE), taxa de desenvolvimento específico (TDE) das pós-larvas de acará-bandeira após 25 dias de experimentação em diferentes densidades de estocagem

\begin{tabular}{cccccc}
\hline \multirow{2}{*}{ Variável } & \multicolumn{5}{c}{ Número de peixes $\mathrm{L}^{-1}$} \\
\cline { 2 - 6 } & 5 & 10 & 15 & 20 & 25 \\
\hline GP $(\mathrm{mg})$ & $0,0409 \mathrm{a}$ & $0,0382 \mathrm{a}$ & $0,0341 \mathrm{ab}$ & $0,0210 \mathrm{c}$ & $0,0246 \mathrm{bc}$ \\
GCT $(\mathrm{mm})$ & $11,16 \mathrm{a}$ & $9,43 \mathrm{ab}$ & $8,47 \mathrm{~b}$ & $7,80 \mathrm{~b}$ & $7,84 \mathrm{~b}$ \\
GCP $(\mathrm{mm})$ & $6,64 \mathrm{a}$ & $5,94 \mathrm{ab}$ & $5,89 \mathrm{ab}$ & $5,20 \mathrm{~b}$ & $5,27 \mathrm{~b}$ \\
GA $(\mathrm{mm})$ & $3,58 \mathrm{a}$ & $2,86 \mathrm{ab}$ & $3,21 \mathrm{ab}$ & $2,37 \mathrm{~b}$ & $2,80 \mathrm{ab}$ \\
TCE $(\%)$ & $13,98 \mathrm{a}$ & $13,67 \mathrm{a}$ & $13,22 \mathrm{ab}$ & $12,05 \mathrm{bc}$ & $11,44 \mathrm{c}$ \\
TDE $(\%)$ & $4,78 \mathrm{a}$ & $4,29 \mathrm{ab}$ & $4,04 \mathrm{~b}$ & $3,84 \mathrm{~b}$ & $3,85 \mathrm{~b}$ \\
\hline
\end{tabular}

Médias com letras diferentes na mesma linha diferem pelo teste Tukey $(\mathrm{P}<0,10)$. 
Os resultados obtidos para GP foram significativamente reduzidos com a utilização de densidades superiores a 15 pós-larvas $\mathrm{L}^{-1}$, sendo os menores resultados, 0,0210 e 0,0246g, obtidos nas densidades mais elevadas 20 e 25 pós-larvas $\mathrm{L}^{-1}$, respectivamente.

De maneira geral, os resultados para ganho entre 0,0409 e $0,0246 \mathrm{~g}$ estão abaixo dos valores encontrados em trabalhos com a mesma espécie. Takahashi (2010), ao estudar o efeito do tipo de alimento no desempenho produtivo com exemplares de peso médio inicial de $0,151 \pm 0,037 \mathrm{~g}$, encontrou valores para ganho de peso entre 0,310 e 0,538g. Volpi et al. (2009), ao estudarem a frequência alimentar, obtiveram resultados ainda maiores, entre 0,46 e $0,91 \mathrm{~g}$, para animais de peso inicial de $3,17 \pm 0,94 \mathrm{~g}$. Os menores valores para GP podem estar relacionados com o tamanho inicial dos animais utilizados no presente trabalho. Foram utilizadas pós-larvas de acará-bandeira recém-eclodidas, com peso médio inicial de 0,00127g, consideravelmente menor que o utilizado pelos demais autores. Contudo, observaram-se elevadas taxas de crescimento específico (Tab. 3).

O aumento da densidade de estocagem afetou negativamente a TCE, sendo observadas as maiores porcentagens de crescimento nos tratamentos com os menores números de animais por unidade experimental. Comparando os valores na faixa de 11,4 e 14,0\% com os demais trabalhos, o presente estudo registra valores elevados para crescimento específico. Tais resultados podem ser explicados pelo fato de peixes jovens apresentarem crescimento mais acelerado em relação a peixes adultos.

Valores para TCE abaixo dos apresentados no presente trabalho são descritos por outros autores. Luna-Figueroa (2003), ao comparar o crescimento inicial de acarás-bandeira alimentados com alimentos vivos (Daphnia e larvas de mosquito) e três rações comerciais para ciclídeos, obteve taxa de crescimento específico que variou entre 4,2 e 6,3\% para animais de peso inicial de 0,06g. Ribeiro (2007) encontrou efeito linear positivo na taxa de crescimento específico de juvenis de acará-bandeira com o aumento do teor de proteína bruta, e o melhor resultado foi de $1,6 \%$ para os animais alimentados com $32,0 \%$ de proteína bruta. Polese et al. (2010), ao avaliarem o efeito de diferentes granulometrias do milho da ração no desempenho de juvenis de pacu, para animais de peso inicial de 8,6g, obtiveram o melhor desempenho com a ração de menor granulometria, sendo $1,14 \%$ o melhor resultado para a taxa de crescimento específico.

As variações nas taxas de crescimento demonstram oscilação de crescimento dos animais e permitem afirmar que as divergências de resultados são atribuídas às diferentes fases do desenvolvimento testadas, sendo a larval uma fase de intensificação do crescimento animal; posteriormente, quanto maior a idade, menor o crescimento.

Ribeiro (2010) ressaltou que o acará-bandeira é vendido por unidade e que seu preço unitário é determinado, principalmente, por classes de tamanho. Portanto, as variáveis relacionadas ao crescimento em comprimento têm maior importância do que as variáveis relacionadas com peso na avaliação de desempenho e no valor comercial dos peixes. Tal crescimento em comprimento pode ser expresso pela TDE que, no presente trabalho, demonstra quanto os animais evoluíram em comprimento durante os dias experimentais.

Nota-se redução na taxa de desenvolvimento específica com o aumento da densidade de estocagem (Tab. 3). O maior resultado foi de $4,78 \%$, obtido para a densidade de 5 pós-larvas $\mathrm{L}^{-1}$, e o menor, de $3,84 \%$, obtido para os animais mantidos na densidade de 20 pós-larvas $\mathrm{L}^{-1}$. Efeito negativo da densidade de estocagem também foi observado para GCT, GCP e GA. Esses valores demonstram que a manutenção dos animais em altas densidades de estocagem pode resultar no prolongamento do período de cultivo destinado ao crescimento, tendo em vista que os animais mais adensados apresentam desenvolvimento letárgico em relação aos animais mantidos em densidades mais adequadas.

As diferenças encontradas no desenvolvimento com o aumento da densidade de estocagem podem ser explicadas pelo aumento da competição e pelo estabelecimento de hierarquia na alimentação entre os peixes. Segundo Vera Cruz e Mair (1994), indivíduos dominantes dentro de uma população podem consumir mais alimento e crescer mais rapidamente, deixando 
menos alimento para os indivíduos submissos, que apresentam menor crescimento. Assim, Degani (1993), para minimizar esse efeito, recomendou a densidade de 0,4 animais $\mathrm{L}^{-1}$, porém referindo-se ao cultivo do peixe até a fase adulta.

Conforme Zuanon et al. (2004) e Berlli (2009), algumas técnicas, como a utilização de aeração suplementar, o aumento de renovação diária da água e a utilização de uma alimentação de boa qualidade, podem ser adotadas para possibilitar a intensificação do cultivo. Tais medidas garantem aumento considerável na densidade de estocagem, sem maiores problemas, relacionada ao desenvolvimento.

Zuanon et al. (2004), ao realizarem um estudo com o objetivo de avaliar o desempenho produtivo de alevinos de tricogaster (Trichogaster trichopterus), submetidos às densidades de $0,05,0,1$, e 0,15 animais $\mathrm{L}^{-1}$, não encontraram efeito da densidade de estocagem no crescimento dos animais. Nagata (2010) não verificou diferenças significativas no ganho de peso e na taxa de crescimento específico do acará-bandeira (Pterophyllum scalare), quando os animais foram submetidos a densidades inferiores a um peixe $\mathrm{L}^{-1}$, com animais de peso médio inicial de $0,80 \pm 0,02 \mathrm{~g}$. Essas respostas podem ser atribuídas às baixas densidades testadas, entretanto os peixes mantidos nas densidades mais elevadas apresentaram os piores resultados de ganho de peso e taxa de crescimento específico, indicando que, possivelmente, a manutenção dos animais por um período prolongado poderá resultar em diferenças maiores.

\section{CONCLUSÕES}

Com base nos resultados obtidos, pode-se inferir que pós-larvas de acará-bandeira podem ser criadas na densidade de 15 pós-larvas $\mathrm{L}^{-1}$, sem terem seu desenvolvimento afetado.

\section{REFERÊNCIAS}

BERLLI, E.L. Estratégia alimentar $e$ desempenho produtivo para acará-disco. 2009. 78f. Dissertação (Doutorando em Ciências Animal) - Escola de Veterinária da Universidade Federal de Goiás, Goiânia.
CACHO, M.S.R.F.; YAMAMOTO, M.E.; CHELLAPPA, S. Comportamento reprodutivo do acará bandeira, Pterophyllum scalare Cuvier e valencien- nes (osteichthyes, Cichlidae). Rev. Bras. Zool., v.16, p.653-664, 1999.

CHAPMAN, F.A.; FITZ-COY, S.A.; THUNBERG, E.M.; ADAMS, C.M. United States of America trade in ornamental fish. $J$. World Aquacu. Society, v.28, p.1-10, 1997.

CHAPMAN, F.A. Ornamental fish culture freshwater. In: STICKNNEY, R.R. (Ed.). Encycopledia of aquaculture, Nova York:Wileyinterscience, p.602-610, 2000.

CRUZ, M.E.P.; SALAS, I.M.; QUEZADAS, H.O. Frecuencia de desove de diferentes variedades del Pez Angel Pterophyllum scalare (Pisces: Cichlidae). Rev. Aquatic., Zaragoza, n.16, [s/p], 2002.

DEGANI, G. Growth and body composition of juveniles of Pterophyllum scalare at different densities and diets. Aquac. Fish. Manag, v.24, p.725-730, 1993.

LUNA-FIGUEROA, J. Pterophyllum scalare (Pisces: Cichlidae): Influência de alimento vivo na reprodução e no crescimento 2003. Disponível em: <http://www.civa2003.org> Acessado em: 07/07/11.

LUZ, R.K. Resistência ao estresse e crescimento de larvas de peixes neotropicais alimentadas com diferentes dietas. Pesq. Agropec. Bras., v.42, p.65-72, 2007.

LUZ, R.K.; ZANIBONI FILHO, E. Larvicultura do mandi-amarelo Pimelodus maculatus Lacépède, 1803 (Siluriformes: Pimelodidae) em diferentes densidades de estocagem nos primeiros dias de vida. Rev. Bras. Zootec., v.31, p.560-565, 2002.

NAGATA, M.M.; TAKAHASHI, L.S.; GIMBO, R.Y. et al. Influência da densidade de estocagem no desempenho produtivo do acará-bandeira (Pterophyllum scalare). Bol. Inst. Pesca, v.36, p.9-16, 2010.

PÉREZ, E.; DIAZ, F.; ESPINA, S. Thermoregulatory behavior and critical thermal limits of angelfish Pterophyllum scalare (Lichtenstein) (Pisces: Cichlidae). J. Therm. Biol., v.28, p.531-537, 2003. 


\section{Gonçalves Júnior et al.}

PEZZATO, L.E.; SCORVO FILHO, J.D. Situação atual da aquicultura na região sudeste. In: VALENTI, W.C. (Ed.) Aquicultura no Brasil: bases para um desenvolvimento sustentável. Brasília: CNPq/Ministério da Ciência e Tecnologia, 2000. p.303-322.

POLESE, M.F.; VIDAL JUNIOR, M.V.; MENDONÇA, P.P. et al. Efeito da granulometria do milho no desempenho de juvenis de pacu, Piaractus mesopotamicus (Holmberg, 1887). Arq. Bras. Med. Vet. Zootec., v.62, p.1469-1477, 2010.

RIBEIRO, F.A. Densidade de estocagem para produção de acará- andeira em viveiros escavados em policultivo com camarão-daamazônia, $R$. Caati., v.23, p.129-134, 2010.

RIBEIRO, F.A.S. Panorama mundial do mercado de peixes ornamentais. Pano. Aquicu., v.18, p.32-37, 2008.

RIBEIRO, F.A.S.; PRETO, B.L.; FERNANDES, J.B.K. Sistemas de criação para o acará-bandeira (Pterophyllum scalare). Acta Scient. Maringá, v.30, p.459-466, 2009.

RIBEIRO, F.A.S.; RODRIGUES, L.A.; FERNANDES, J.B.K. Desempenho de juvenis de acará-bandeira (Pterophyllum scalare) com diferentes níveis de proteína bruta na dieta. $B$. Inst. Pesca, v.33, p.195-203, 2007.

SILVA, S.D; MENDES, G.N.; VALENÇA, A.R. Cultivo de pós-larvas de Macrobrachium rosenbergii (De Man, 1879) com os alevinos de Pterophyllum scalare (Heckel, 1840) e Carassius auratus (Günther, 1870) em laboratório. B. Inst. Pesca, São Paulo, v.34, p.453 - 461, 2008.

TAKAHASHI, L.S.; SILVA, T.V.; FERNANDES, J.B.K. et al. Efeito do tipo de alimento no desempenho produtivo de juvenis de acarábandeira (Pterophyllum scalare). B. Inst. Pesca, São Paulo, v.36, p.1-8, 2010.

VERA CRUZ, E.M.; MAIR, G.C. Conditions for efective androgen sex-reversal in Oreochromis niloticus. Aquac., v.122, p.237-248, 1994.

VOLPI, G.C.L.; HA, N.; TOMOMI, J.K. et al. Efeito da frequência alimentar no desempenho produtivo de acará-bandeira (Pterophyllum scalare). In: SIMPÓSIO DE CIÊNCIAS DA UNESP - DRACENA, 5., 2009, Dracena. (Resumo expandido).

ZUANON, J.A.S.; ASSANO, M.; FERNANDES, J.B.K. Desempenho de tricogaster (Trichogaster trichopterus) submetido a diferentes níveis de arraçoamento e densidades de estocagem. Rev. Bras. Zootec., v.33, p.1639-1645, 2004. 